\title{
Reflets
}

Revue ontaroise d'intervention sociale et communautaire

\section{Image corporelle et minceur : à la poursuite d'un idéal élusif}

\section{Sophie Vinette}

Volume 7, numéro 1, printemps 2001

Santé mentale et les défis de l’an 2001

URI : https://id.erudit.org/iderudit/026340ar

DOI : https://doi.org/10.7202/026340ar

Aller au sommaire du numéro

Éditeur(s)

Reflets : Revue ontaroise d'intervention sociale et communautaire

ISSN

1203-4576 (imprimé)

1712-8498 (numérique)

Découvrir la revue

Citer cet article

Vinette, S. (2001). Image corporelle et minceur : à la poursuite d'un idéal élusif. Reflets, 7(1), 129-151. https://doi.org/10.7202/026340ar

Tous droits réservés (C) Reflets : Revue ontaroise d'intervention sociale et communautaire, 2001
Ce document est protégé par la loi sur le droit d'auteur. L'utilisation des services d'Érudit (y compris la reproduction) est assujettie à sa politique d'utilisation que vous pouvez consulter en ligne.

https://apropos.erudit.org/fr/usagers/politique-dutilisation/ 


\title{
Image corporelle et minceur : à la poursuite d'un idéal élusif ${ }^{1}$
}

\author{
Sophie Vinette \\ Maîtrise en service social, Université d'Ottawa
}

\section{Introduction}

Il semble que depuis toujours, les femmes interrogent l'image que leur renvoie le miroir. Pourtant, le reflet nait davantage du regard que de la glace. Peu importe son poids et sa taille, il faut à tout prix se soucier de son apparence, veiller à l'améliorer, à la transformer et à l'entretenir, ce qui nécessite toute une discipline et implique plusieurs coûts, que ce soit en termes de temps, d'argent ou d'énergie. Même en l'absence de tout excès de poids ou en présence d'une silhouette correspondant parfaitement au modèle valorisé, la population féminine fait l'objet de régulation et de surveillance - que ces dernières soient auto-imposées ou qu'elles proviennent de l'extérieur - afin de ne pas déraper de l'idéal, ou encore de s'assurer que la quête interminable de l'image se poursuive.

Comment expliquer les résultats des recherches réalisées en Amérique du Nord et en Europe qui indiquent que plus de $90 \%$ des femmes se perçoivent trop grosses? D'où dérive cette profonde insatisfaction de l'image corporelle? Cet article propose justement d'explorer cette problématique en définissant d'abord en quoi consiste l'image corporelle et en dressant un portrait de la prévalence de l'insatisfaction de l'image corporelle et des désordres alimentaires auprès de la population féminine. Les significations accordées à la minceur et au gras, de même que les répercussions qu'entraîne la 
poursuite de l'idéal de la minceur sur la santé seront ensuite mises en lumière. Par ailleurs, il sera également question d'illustrer la construction sociale du diagnostic de l'anorexie et les barrières associées à l'apparence. Enfin, une lecture plus poussée du corps et de l'anorexie sera reprise en introduisant la notion de pouvoir.

\section{Des chiffres qui en disent long}

L'image corporelle est un concept auquel se réferent plusieurs auteurs lorsqu'ils examinent la relation problématique qu'entretiennent les femmes face à leur corps. Bien que superficielle, l'apparence physique est déterminante dans les jugements qui sont portés sur notre personne et l'impression qui s'y dégage. Selon Le Breton (1997: 96),

l'apparence corporelle répond à une mise en scène par l'acteur, touchant la manière de se présenter et de se représenter. Elle englobe la tenue vestimentaire, la manière de se coifferet d'apprêter son visage, de soigner son corps, etc., c'est-à-dire un mode quotidien de se mettre socialement en jeu, selon les circonstances, à travers une manière de se montrer et un style de présence.

Bien qu'une foule d'éléments contribuent à définir l'apparence physique, le poids ou la perception de son poids est probablement le facteur prédominant dans la détermination de l'image corporelle chez les femmes (Davis 1997). L'image corporelle comprend à la fois les aspects cognitifs - comme les perceptions que l'on entretient à l'égard de notre corps et les expériences corporelles, - et les aspects émotionnels concernant le plaisir ou le déplaisir, et la satisfaction ou l'insatisfaction que l'on ressent face à l'apparence du corps et à ses fonctions (Pruzinsky et Cash 1990, dans Davis 1997). Il importe également de noter que ces cognitions et ces émotions sont purement subjectives, et peuvent donc se révéler très près de la réalité ou encore très éloignées de celle-ci (Davis 1997). S'il est vrai que nous vivons à travers le regard des 
autres et que notre image se reflète dans leurs yeux (Bideaud 1991, dans Travaillot 1998), alors nous risquons tous d'être façonnés au gré des gens qui nous entourent. C'est donc dire que «la mise en scène de l'apparence livre l'acteur au regard évaluatif de l'autre, et notamment à la pente du préjugé» (Le Breton 1997: 97). Les critères de perfection corporelle socialement imposés fournissent ainsi les cadres sur lesquels viennent se modeler les cognitions que nous entretenons, et les émotions que nous éprouvons, face à notre corps. L'image que nous avons de notre corps est clairement instrumentale dans la création de nos comportements et de nos attitudes. De même, cette image peut également se transformer par certains comportements (Davis 1997). Somme toute, il importe de se rappeler que l'image corporelle d'une personne n'est pas déterminée par la silhouette et la taille actuelles de son corps, mais par l'évaluation subjective que cette personne fait de ce que signifie avoir un tel type de corps à l'intérieur d'une culture particulière (Grogan 1999).

À ce titre, les revues, le cinéma et la télévision de même que les affiches publicitaires sont souvent invoqués pour expliquer les préoccupations incessantes des femmes devant la minceur (Genest 1996). Ceux-ci émettent des critères de beauté féminine difficiles, voire impossibles à atteindre. Le contexte actuel fait en sorte que les hommes sont jugés surtout en termes de leurs accomplissements et de leurs performances, alors que c'est l'apparence qui témoigne de la désirabilité et de la valeur des femmes (Brownmiller 1984, dans Paludi 1998). Or, en tentant d'adhérer aux définitions biaisées et irréalistes des critères esthétiques, les femmes posent de sérieux risques à leur santé (Jackson 1992, Smith 1966, dans Paludi 1998). Cette poursuite de la minceur - et les effets qu'elle entraîne ne se limite toutefois pas aux femmes adultes; elle se manifeste de manière flagrante chez les adolescentes et de plus en plus chez les fillettes. En effet, l'Institut national de la nutrition du Canada affirme que le tiers des femmes qui affiche un poids santé sont présentement au régime, alors que les jeunes filles commencent dès l'âge de 9 ou 10 ans à suivre cet exemple (Guéricolas 1998). Wolf (1990) relate d'ailleurs que plus de $80 \%$ des fillettes de 10 ans suivent un régime. Tout comme les pratiques diététiques, 
l'insatisfaction de l'image corporelle est un phénomène qui se répercute chez les femmes de tout âge. À ce titre, une étude rapporte que $53 \%$ des adolescentes de 13 ans se montraient insatisfaites de leur corps alors que ce pourcentage grimpait à $78 \%$ chez les adolescentes de 17 ans (Jacobs Brumberg 1997). Du côté des jeunes filles, un sondage effectué auprès de 494 fillettes âgées de 9 et 10 ans indique que plus de $50 \%$ de celles-ci se décrivaient obèses, bien que seulement $15 \%$ d'entre elles l'étaient effectivement selon les critères médicaux (Wolf 1990). Dans la même veine, une autre étude révèle qu'à l'instar des femmes adultes, des fillettes aussi jeunes que 8 ans se montraient insatisfaites de leur image corporelle, ce qui suggère la tentative de se mouler, mais avec grande difficulté, à l'idéal de la minceur (Grogan et Wainwright 1996). Sachant que l'adhésion de la population féminine à cet idéal s'accompagne d'une augmentation de la prévalence des désordres alimentaires et des perturbations de l'image corporelle (Lamb et al. 1993), il y a lieu de s'inquiéter.

Le Centre national d'information sur les désordres alimentaires confirme que plus de 300000 Canadiennes sont affligées d'un trouble alimentaire, ce qui représente au moins $7 \%$ de la population féminine. Tout aussi alarmant est le taux de mortalité qui varie de $10 \%$ (Paludi 1998) à $15 \%$ (Poulton 1996). D'autres études révèlent que la prévalence des symptômes associés aux désordres alimentaires se situent entre $5 \%$ et $15 \%$ chez les étudiantes de niveaux collégial et secondaire (Nagel et Jones 1992, dans Austin 1999). Approximativement $1 \%$ de toutes les femmes âgées entre 12 et 25 ans souffrent d'anorexie mentale alors que le ratio femmes-hommes se situe à 20:1 (Paludi 1998). Pour sa part, la boulimie semble être un problème relativement répandu, mais tout aussi redoutable, au sein des populations collégiales; les sondages effectués révèlent un taux d'incidence qui varie entre $13 \%$ et $67 \%$ chez les femmes pré-diplômées (Paludi 1998).

Tout compte fait, les statistiques illustrent de façon assez explicite l'ampleur du phénomène et les personnes qui risquent le plus d'être touchées par celui-ci. Par contre, il reste encore beaucoup à connaître sur le pourquoi des manifestations de cette problématique, et sur les enjeux qui y sont rattachés. La tendance 
qu'ont l'insatisfaction de l'image corporelle, la valorisation de la minceur et le développement des désordres alimentaires à s'afficher dans les sociétés d'abondance, et davantage auprès de la population féminine, n'est pas issue du hasard.

\section{Une image vaut mille mots}

Pour être en mesure de mieux comprendre les points d'ancrage dans laquelle s'insère la problématique entourant l'insatisfaction de l'image corporelle, il importe de saisir les significations accordées à un corps mince et à un corps gras. Ainsi, dans les sociétés occidentales affluentes, la minceur est synonyme de modération, de maitrise de soi (Hesse-Biber 1996), de succès et d'acceptation sociale (Grogan 1999) alors que l'obésité est considérée comme un échec moral, une incapacité à retarder la satisfaction et à se contrôler (Hesse-Biber 1996), un manque de discipline ou encore une tendance à la paresse (Bordo 1990; Grogan 1999) et à l'avidité (Sparkes 1997). On parle ainsi fréquemment de l'indulgence et du manque de volonté des personnes obèses qui mangent «trop» ou qui ne s'adonnent pas à des exercices physiques. Dans cette culture de consommation où les médias populaires mettent constamment l'accent sur les bénéfices esthétiques du maintien du corps, les individus qui parviennent à faire correspondre leur corps aux images idéalisées de la jeunesse, de la santé, de la bonne forme physique et de la beauté possèdent une valeur économique d'échange plus élevée que ceux et celles qui ne peuvent, ou qui ne désirent pas s'approprier de telles images (Featherstone 1991, dans Sparkes 1997). Puisque l'excès de poids est une caractéristique perçue négativement dans la société nord-américaine (Dyrenforth et al. 1980), les femmes dont le corps correspond aux normes esthétiques valorisées se voient en meilleure mesure d'assurer leur ascension sociale (Guéricolas 1998). La silhouette est ainsi devenue une indication critique de la valeur personnelle des gens, le corps pouvant se présenter comme un symbole de santé, de succès et de richesse, ou encore être jugé tout à fait à l'opposé. 
À ce titre, Austin (1999) offre un regard critique intéressant sur le rôle pathogénique joué par la science, la technologie et la santé publique dans la création d'une culture où les relations entre la nourriture et le gras sont désordonnées. En s'appuyant sur les ouvrages de Kleinman, Bordo et Foucault, l'auteur illustre comment les préoccupations concernant la nourriture, le gras et les diètes sont non seulement devenues des normes dans notre culture mais également l'apanage des promoteurs de la santé publique qui encouragent la formation et le maintien de tels soucis.

Or, le discours produit par la santé publique, et que cette dernière se plaît à présenter sous la rubrique de la nutrition, s'appuie en fait sur une perspective biomédicale matérialiste et sur des idéologies basées sur la déviance et la différence (Austin 1999). Turner (1992, dans Austin 1999) identifie d'ailleurs un lien temporel entre le développement du caractère scientifique accordé à la nutrition et l'émergence de représentations du corps en tant que machines dont les besoins et les dépenses énergétiques peuvent être mathématiquement quantifiés, d'où l'idée de représenter la nourriture en terme de calories.

Les efforts acharnés destinés à la maximisation de la forme physique et à l'évitement de l'obésité ne seraient pas tant liés au désir de préserver une bonne santé qu'au besoin d'afficher une apparence extérieure attrayante (Lupton 1994). Dans le but de promouvoir la consommation, les industries qui encouragent les activités physiques et la santé font naître l'incertitude entourant l'apparence, nourrissant du même coup l'anxiété face à l'image corporelle (Freund et Fisher 1982).

Les campagnes actuelles de promotion de la santé mettent ainsi l'accent sur l'individu en associant plusieurs maladies avec son mode de vie et ses comportements. Le raisonnement derrière ces campagnes repose sur l'idée selon laquelle l'incidence des maladies sera réduite si les membres de la population peuvent être persuadés qu'ils sont en mesure d'exercer un plus grand contrôle sur leurs affections corporelles (Sparkes 1997). À titre d'exemple, la plupart des gens en viennent à assumer qu'ils peuvent effectivement contrôler leur poids et, conséquemment, réduire l'incidence de la maladie en surveillant leur apport 
calorique (Bennett 1984, dans Austin 1999). Cette conviction reflète une perception très répandue dans notre culture voulant que le contrôle intentionnel de l'apport calorique soit normal ou désirable, et que l'auto-restriction alimentaire soit nécessaire et suffisante pour maintenir un dépôt adéquat des tissus adipeux. Le corps en vient ainsi à être considéré comme une machine pouvant fonctionner au gré des manipulations du machiniste (Turner 1992, dans Austin 1999).

La santé, pour sa part, devient un but en soi qui ne peut s'accomplir que par des actions intentionnelles, ces dernières impliquant la maîtrise de soi, la persévérance et l'engagement en termes de temps et d'énergie. Le poids constitue le symbole par excellence de la maîtrise de soi, un corps mince témoignant du contrôle, et un corps souffrant d'embonpoint étant la preuve d'une indulgence exagérée et d'un manque de volonté (Lupton 1994). Crawford (1984, dans Lupton 1994) fait ainsi le lien entre ces correspondances et les discours de la responsabilité individuelle et du contrôle de soi qui prédominent la rhétorique de la promotion de la santé. Selon cet auteur, la "santé» est un discours moral qui intègre l'importance accordée à l'individualisme, au travail ardu et aux récompenses matérielles dans la culture contemporaine nordaméricaine. À ce titre, la recherche de Saltonstall (1993, dans Lupton 1994) confirme les impressions de Crawford, la santé étant vue par les participantes et les participants de l'étude comme un accomplissement personnel pouvant être atteint par des actions délibérées et intentionnelles comme l'exercice physique, les régimes alimentaires et un horaire de sommeil suffisant. Une différence majeure existait toutefois entre les femmes et les hommes interviewés.Alors que les hommes avaient tendance à se voir maittres de leur corps et à y appartenir, les femmes faisaient plutôt référence aux difficultés à contrôler un corps récalcitrant, particulièrement lorsqu'il s'agissait des pratiques diététiques (Lupton 1994). En d'autres termes, les hommes valorisaient l'entretien du corps parce qu'il leur permettait de maximiser leur potentiel d'action alors que pour les femmes, ce contrôle avait l'effet contraire, les obligeant ainsi à se préoccuper de leur apparence physique du fait qu'elle ne correspondait pas aux canons esthétiques acceptés. 


\section{De lourdes conséquences sur la santé}

De toute évidence, le poids semble contribuer grandement à définir la valeur et l'identité des femmes (Lamb et al. 1993). Cette poursuite de l'idéal de la minceur n'est toutefois pas sans répercussions désolantes. Étant donné la difficulté qu'éprouvent les femmes à se mouler au modèle irréaliste valorisé, elles sont souvent contraintes à recourir à des pratiques qui s'avèrent destructives à leur santé et à leur estime de soi (Hesse-Biber 1996).

Et qui de mieux placé pour nourrir et exacerber ces appréhensions face au corps que les marchés en pleine croissance qui renouvellent sans cesse «les signes visant à l'entretien et à la mise en valeur de l'apparence sous les auspices de la séduction?» (Le Breton 1997: 98). Plusieurs industries profitent donc de l'insécurité des femmes concernant leur apparence physique en nourrissant, par la diffusion exacerbée de l'idéal de la minceur, l'illusion qu'il est possible «de modeler son cops à l'infini (...) et de transformer sa nature première» (Travaillot 1998: 149). Conséquemment, l'industrie de la chirurgie esthétique, dont $94 \%$ de sa clientèle est composée de femmes, effectue des recettes annuelles de l'ordre de 5 milliards de dollars (Hesse-Biber 1996). De son côté,les industries des produits et des services reliés à la perte de poids réalisent d'énormes profits, les ventes annuelles s'élevant à environ 3 milliards au Canada, et à plus de 40 milliards aux États-Unis (Poulton 1996).

Les industries rattachées à la perte de poids illustrent d'ailleurs de façon frappante l'intersection des structures médicales, économiques et politiques qui gardent les femmes dans un état constant de «maladie» (Spitzack 1990). Il faut comprendre qu'un poids corporel approprié, tel que déterminé par les nombreux discours, représente seulement une partie d'un système normatif qui apparie santé, apparence féminine et comportement. Les éléments de ce système se recoupent et se consolident l'un l'autre pour accomplir deux buts. Le premier amène les femmes à dépenser des sommes d'argent considérables en vue de préserver leur apparence et leur santé, permettant ainsi aux structures 
économiques capitalistes de subsister. Le corps féminin est donc un bien qui nécessite l'achat d'innombrables produits pour conserver sa valeur. En deuxième lieu, les femmes prennent part au renforcement de l'imagerie féminine à l'intérieur de la culture en s'inclinant de manière ostensible au perpétuel regard des autres (Spitzack 1990).Toutefois, comme Bourdieu (1995, dans Travaillot 1998: 217) le soulève, il est important de reconnaitre que :

la domination masculine, qui constitue la femme en objet symbolique, dont l'être est un être-perçu, a pour effet de placer les femmes dans un état permanent d'insécurité corporelle, ou mieux, d'aliénation symbolique. Dotées d'un être qui est paraître, elles sont tacitement sommées de manifester, par leur manière de tenir leur corps et de le présenter (vêtements, maquillage, tenue, etc.) une sorte de disponibilité (sexuée et éventuellement sexuelle) à l'égard des hommes.

De toute évidence, les discours en tant que pratiques sociales ont de puissants effets puisqu'ils régularisent les sujets tout en définissant ce qui est considéré normal et anormal pour différents groupes d'individus dans une variété de contextes sociaux (Malson 1997). En faisant la lumière sur les répercussions institutionnelles, sociales et culturelles du système médical, Illich (1992) met aussi en relief la médicalisation du social et le culte de la santé. Ainsi, dans le processus de médicalisation du corps des femmes, les aspects sociaux sont occultés au profit d'une vision scientifique apparemment objective (Robertson 1992; Blais 1993). Par conséquent, la «maladie» tombe sous l'égide de la science médicale, cette dernière participant non seulement à la construction du corps des femmes mais imposant également ses connaissances à partir des définitions qu'elle en fait. Au cours de ce processus de médicalisation, le corps féminin devient l'objet d'un regard et d'un discours médical qui le reconstitue comme un corps tributaire des critères normatifs médicaux qui, eux, émanent de principaux médicaux universels (Robertson 1992). Nul doute, il s'ensuit une conceptualisation très restreinte et fermée du corps. À cet effet, Zola (1992 : 46) rapporte que «dès qu'un problème est identifié 
comme une maladie, il n'est plus, par définition, social et on ne s'attend plus à ce que les mesures correctives soient sociales». Par conséquent, les personnes sont considérées comme les porteuses du problème qu'elles manifestent alors que la communauté ou la société s'abdique de porter le fardeau de la responsabilité pour son apparition et l'élaboration d'une solution (Zola 1992).

\section{Construction du diagnostic de l'anorexie}

Un exemple lié à la problématique dont il est question ici suffira à illustrer comment le système médical, au sens large du terme, évase ses fonctions initiales par le biais d'un discours «normalisateur et moralisateur» (Travaillot 1998 :218). En s'appuyant sur la perspective du constructivisme social, il semblerait que les discours médical et psychologique ne décrivent pas simplement de manière plus ou moins objective «l'anorexie mentale», mais construisent plutôt sa définition de manière particulière (Foucault 1972,1979, dans Malson 1997). «L'anorexie mentale» est conçue comme une entité distincte causant plusieurs des problèmes que vivent les femmes «anorexiques», ce qui obscurcit l'idée selon laquelle l'anorexie mentale est en fait une étiquette diagnostique socialement construite ayant des connotations et des conséquences particulières pour les fillettes et les femmes (Malson 1997). Non seulement cette perspective institutionnelle de l'anorexie forme notre compréhension de l'expérience des femmes et des jeunes filles, mais elle régularise également ces expériences. De tels discours ont donc tendance à produire des connaissances universelles en réduisant ou en ignorant complètement la diversité des expériences des femmes et des jeunes filles diagnostiquées comme anorexiques, d'où l'homogénéisation d'une catégorie de maladie (Malson 1997).

Les compagnies d'assurance, les industries pharmaceutiques et les cliniques d'obésité participent également à ce discours médicalisant en établissant des chartes de poids normatives, en mettant sur le marché des médicaments de toutes sortes pour couper l'appétit et en injectant leurs patientes d'hormones diverses 
afin de favoriser la perte de poids. Or, plutôt que d'améliorer la santé des femmes, ces pratiques risquent de la compromettre (Daigneault-L'Archevêque et al. 1991). Par ailleurs, comme le soulève Millman (1981, dans Spitzack 1990), les solutions avancées pour enrayer l'obésité sont souvent plus dangereuses que la «maladie» elle-même, le fait de perdre et de gagner du poids de manière répétée posant davantage de risques à la santé que le fait de rester à un poids légèrement supérieur à la «normale». Plusieurs contradictions émergent donc du discours médical, notamment l'idée selon laquelle le traitement idéal contre l'obésité repose sur les régimes alors que cette pratique se veut l'une des causes principales du regain de poids (Daigneault-L'Archevêque et al. 1991). Les «solutions» mises de l'avant cherchent donc beaucoup plus à générer des profits qu'à guérir l'obésité (Spitzack 1990).

Enfin, Robertson (1992) précise qu'à la fois les discours médical et profane font correspondre la féminité à la retenue et à la maitrise de soi. Celles qui se privent excessivement - comme les anorexiques - et celles qui de toute évidence n'exhibent pas suffisamment de contrôle - comme les personnes obèses deviennent l'objet d'admiration ou de dégoût; ces réactions permettent à leur tour de façonner la signification et l'identité que la personne entretiendra dans sa relation avec la nourriture (Robertson 1992). Les pressions risquent ainsi de se faire particulièrement intenses à l'endroit des membres de la société qui sont déjà susceptibles d'être marginalisés au niveau de l'acceptation et du pouvoir: les minorités visibles, les femmes et les adolescentes (Dyrenforth et al. 1980).

\section{Contraintes associées à l'apparence}

La valorisation de la jeunesse (Zola 1992), le niveau socioéconomique et l'appartenance raciale ou ethnique (Beausoleil 2000) représentent tous des barrières sociales qui perpétuent l'effet d'exclusion. L'une des contraintes les plus souvent associées à l'apparence des femmes a trait à l'âge. En effet, l'âge constitue 
pour la population féminine une barrière dans plusieurs sphères de la vie quotidienne, comme sur les plans professionnel et relationnel. La grande variété de produits amaigrissants, esthétiques et liés au conditionnement physique qui sont actuellement créés, vantés et vendus témoignent de l'importance que revêt l'apparence et la conservation d'un corps jeune et attrayant dans cette société capitaliste qui encourage les individus à adopter des stratégies instrumentales pour lutter contre la détérioration et l'affaissement (Featherstone 1991, dans Sparkes 1997).

Les études qui se sont intéressées à la satisfaction de l'image corporelle à travers le cycle de vie révèlent que les femmes de tout âge sont moins satisfaites que les hommes, bien qu'elles ne paraissent pas devenir moins satisfaites à mesure qu'elles vieillissent (étant donné que leur idéal semble devenir plus massif en vieillissant, c'est-à-dire davantage semblable à leur silhouette actuelle) (Grogan 1999).

Même si elles ont fait l'objet de moins d'études, il existe également d'autres barrières associées à l'apparence qui méritent d'être mentionnées, comme le niveau socioéconomique et la race. La pauvreté, par exemple, limite fréquemment les choix des femmes de classe défavorisée en ce qui a trait à leurs pratiques alimentaires et vestimentaires, augmentant du même coup les risques de stigmatisation rattachés à la pauvreté. Les préoccupations des femmes provenant de milieux défavorisées ne reposeraient pas uniquement sur la présentation de soi au niveau de la minceur mais s'attarderaient également à l'élaboration de stratégies visant à réduire les risques de stigmatisation rattachés à la pauvreté, un statut qu'elles veulent cacher pour éviter qu'on le lise sur leur corps. De toute évidence, les femmes de classe moyenne ou supérieure évoquent différentes préoccupations, distinctions et stratégies en ce qui a trait à leur image corporelle, de sorte que l'évaluation de l'apparence se verra influencée par la classe sociale à laquelle la personne appartient. L'apparence des femmes de la classe ouvrière risque ainsi d'être jugée plus sévèrement que celle des membres de la classe dominante, la paresse ou l'inactivité étant souvent soulevées pour expliquer les rondeurs qu'elles affichent (Beausoleil 2000). 
Finalement, en termes de caractéristiques de la race ou de l'ethnicité, les femmes qui ne font pas partie de la culture blanche dominante, mais qui aspirent à la présentation d'une image correspondante aux normes de beauté risquent d'être doublement marginalisées. Historiquement - et encore aujourd'hui —, la population féminine blanche de classe moyenne ou supérieure se veut le modèle dominant de beauté et de féminité dans la société occidentale contemporaine. Conséquemment, les femmes qui échappent à de telles caractéristiques sont par définition exclues des canons esthétiques dominants et, par extension, deviennent «autres», ou encore «déviantes» (Collins 1990, Hooks 1992, Bordo 1993, dans Beausoleil 2000). Les critères de beauté valorisés qui sont associés à la culture occidentale dominante finissent par isoler et sexualiser simultanément les femmes qui ne sont pas blanches (Clarke Hine 1990, Collins 1990, Hooks 1992, 1993, dans Beausoleil 2000; Bourdieu 1979). À travers l'apparence, les personnes caucasiennes "définissent, jugent, "racialisent" et sexualisent les "autres" femmes» (Beausoleil 2000 : 243). Par la force et l'étendue des discours dominants, ces dernières ont la nette impression qu'elles doivent accorder une attention particulière à leur apparence physique (Beausoleil 2000) si elles souhaitent se rapprocher de l'image idéalisée.

Il appert donc que les femmes qui ne sont pas de race blanche et qui proviennent de milieux défavorisés - mais qui aspirent aux normes esthétiques valorisées par la culture occidentale dominante ou qui s'identifient à celle-ci — risquent d'être jugées encore plus sévèrement et, par conséquent, sont davantage susceptibles de se montrer insatisfaites de leur image corporelle et de se sentir exclues ou marginalisées.

De toute évidence, la marge de manoeuvre est très mince pour quiconque désire aspirer aux idéaux présentés par la société occidentale, mais particulièrement pour les femmes qui n'ont guère d'espace pour créer une féminité acceptable selon les normes dominantes de la suprématie blanche. Qui plus est, ces tensions autour de l'apparence sont non seulement observables entre diverses cultures mais se veulent également interethniques (Beausoleil 2000), la personne étant parfois tiraillée entre son désir 
de correspondre aux normes dominantes et de se sentir acceptée, et entre son devoir de reconnaitre et de valoriser son héritage culturel.

Bref, les résultats obtenus en lien avec l'image corporelle qu'affichent les gens de classe, d'âge, de sexualité et de groupe ethnique différents soulignent l'importance cruciale de comprendre les pressions socioculturelles qui s'exercent sur ces membres pour être en mesure de saisir le sens des variations notées au niveau de l'image corporelle (Grogan 1999). Compte tenu des nombreux sondages et recherches qui confirment l'insatisfaction qu'entretiennent les femmes de tout âge face à leur image corporelle - et en particulier à leur poids - et les répercussions négatives qui en découlent, il devient évident que ce phénomène mérite un examen plus approfondi.

\section{Corps et anorexie mentale}

Le corps - ce que nous mangeons, comment nous nous vêtons, les rituels quotidiens à travers desquels nous en prenons soin - est un médium de la culture. Il est non seulement, comme nous l'avons vu, une forme symbolique puissante, mais il est aussi, comme le soutiennent Bourdieu (1977) et Foucault (1976), un lieu pratique et direct de contrôle social. Banalement, à travers des routines qui peuvent nous paraitre dérisoires, des habitudes, des règles et des pratiques, la culture s'inscrit sur le corps et devient une activité automatique et habituelle. Les corps «régularisés» sont donc issus de pratiques culturelles et s'habituent aux règles de cette vie culturelle (Bordo 1989).

Dans l'analyse de l'insatisfaction de l'image corporelle, il s'avère utile de recourir à ces deux conceptions du corps, c'est-à-dire au corps en tant que surface sur laquelle le social s'inscrit,et au corps en tant que site de l'expérience vécue (Grosz 1992, dans Frigon 2000). Même si les femmes ont tendance à être "perçues comme étant inextricablement liées à leur corps, voire emprisonnées par 
lui» (Frigon 2000 : 154), le corps doit être vu à la fois comme un lieu de contrôle et un lieu de résistance. Puisque l'enjeu du pouvoir dans les institutions repose - directement ou indirectement sur l'emprise au corps (Préjean 1994, dans Frigon 2000), il semble important d'examiner comment le corps en vient à être façonné, marqué et incarné à travers les pratiques qu'on lui assigne et qu'on lui impose, ce qui nous amène à l'exploration de l'anorexie mentale.

Bien qu'un regard tourné vers le passé témoigne de la présence de cas d'anorexie au $19^{\mathrm{e}}$ siècle, le phénomène des désordres alimentaires sur une échelle massive est unique à la culture des années 80 comme l'épidémie de l'hystérie l'a été pour l'ère victorienne (Bordo 1989). Que l'on s'attarde à l'hystérie ou à l'anorexie, Bordo (1989) trouve que dans les deux cas, le corps de la personne souffrante est profondément empreint d'une construction idéologique de la féminité emblématique des périodes en question. Cette construction est de toute évidence toujours homogénéisante et normalisatrice, effaçant, entre autres, les différences raciales et de classes, et insistant sur l'idée que toutes les femmes aspirent à un idéal coercitif standardisé (Bordo 1989). Désormais, on ne nous dit plus ce qu'est une «femme» ou en quoi consiste la féminité; nous apprenons plutôt les règles directement à travers les discours corporels, c'est-à-dire à travers des images qui nous disent quels vêtements, quelle silhouette, quelle expression faciale, quels mouvements et quels comportements sont requis (Bordo 1990).

Par ailleurs, Bordo (1999) est d'avis que les désordres alimentaires n'ont pas seulement à voir avec les nouvelles attentes sociales face aux femmes et l'ambivalence envers leur corps, mais paraissent également liés à des inquiétudes plus générales en ce qui a trait au corps comme source de besoins et de vulnérabilités physiques hors de notre contrôle. Parmi les culturistes et les personnes souffrant d'anorexie, il existe les mêmes conceptualisations déconcertantes du corps comme étranger. Nous y retrouvons également la même emphase sur le contrôle, c'est-àdire sur l'impression que leur vie est fondamentalement hors de contrôle, et sur le sentiment d'accomplissement qui émane de la 
maîtrise totale de leur corps (Bordo 1988). Ce sentiment de maitrise semble provenir de deux sources : (1) d'abord, il y a la croyance rassurante que quiconque peut vaincre tout obstacle physique; et (2) il y a l'impression d'être complètement en charge de la forme de son corps, l'individu pouvant créer des oeuvres d'art en «sculptant» son corps. Le sentiment de sécurité qui découle de l'accomplissement d'un tel objectif semble être lié au fait que les gens ne peuvent désormais plus contrôler les événements extérieurs à eux-mêmes - comment ils réussissent au travail et s'en tirent dans leurs relations personnelles, par exemple - mais ils peuvent maitriser la nourriture qu'ils ingèrent et la distance qu'ils parcourent. L'abstinence (jeûne ou faible consommation de nourriture) et les tests d'endurance (exercices physiques acharnés) sont des moyens de prouver leur autosuffisance dans une culture où la survie est souvent à la merci de spécialistes, d'experts, de machines et de technologies sophistiquées, et où le corps se prête à la dépendance et à la vulnérabilité (Bordo 1988).

Enfin, les désordres alimentaires seraient également associés aux contradictions émanant de la culture de consommation qui encourage continuellement les individus à satisfaire leurs désirs mais qui valorise simultanément l'autodiscipline et méprise le gras en faisant rimer ce dernier avec paresse et manque de volonté (Bordo 1999). La minceur, qui est, comme nous l'avons vu, synonyme de compétence, d'autocontrôle et d'intelligence, devient une manifestation de l'endiguement des impulsions et du triomphe de la volonté sur le corps (Lester 1997).

Néanmoins, il est paradoxal de constater qu'au niveau culturel, le corps féminin mince communique presque l'opposé de ce qu'il signifie au niveau psychologique - la conformité à des stéréotypes sexuels, la vanité, la superficialité, le besoin d'acceptation et d'approbation, la vulnérabilité, la délicatesse et la fragilité, de même qu'un désir de prendre le moins d'espace (physique/social) possible (Lester 1997). La femme souffrant d'anorexie se trouve donc prise dans une situation paradoxale où le chemin emprunté pour sa libération - la minceur - est celui qui l'asservit encore plus profondément. Ainsi, pour Beaulieu (1988), les désordres alimentaires traduisent le plus souvent une souffrance de l'âme, un mal 
de vivre, qui se répercute dans le corps. «Le corps, devenu méprisable, représente une blessure permanente» ( $\left(\mathrm{D}^{\mathrm{r}}\right.$ Bigras, dans Beaulieu 1988: 177). L'anorexie semblerait donc plutôt découler de la réalisation affligeante que «je suis mon corps» dans cette culture où les corps définissent qui nous sommes, quelles opportunités se présenteront ou seront révoquées, quelles seront nos expériences et comment les autres se comporteront avec nous (Lester 1997). En ce sens, la poursuite incessante de l'extrême minceur peut s'avérer une tentative pour incarner certaines valeurs, pour créer un corps qui parlera de lui-même d'une manière significative et puissante (Bordo 1993b; Weiss 1999).

Les liens tissés entre les concepts abordés et le développement de troubles alimentaires soulèvent des relations complexes qu'une seule perspective ou qu'une série de travaux ne parviendraient à traduire adéquatement ni complètement. Quoique certaines théories gagnent en popularité et se révèlent des plus intéressantes, il peut s'avèrer tentant de tomber dans le piège de la facilité dans la mesure où les explications offertes correspondent intuitivement à notre compréhension du problème, circonscrivant du même coup tout questionnement ultérieur. Par exemple, de nombreux travaux examinant l'anorexie et préconisant une perspective socioculturelle sont limités à deux niveaux. En premier lieu, il existe une tendance à focaliser uniquement sur les pressions sociales pour «maigrir» et atteindre une apparence physique «élégamment mince» à l'exclusion d'autres facteurs socioculturels pouvant se révéler pertinents (Malson 1997). Selon plusieurs sociologues et anthropologues, l'image de l'anorexie comme un problème de développement transitoire et auto-infligé par des jeunes femmes perdues dans leur monde de la mode et de restriction calorique constitue un stéréotype réducteur pouvant occulter les préoccupations réelles des femmes. De plus, en mettant l'accent sur la minceur, l'idée centrale que l'on se fait des préoccupations liées à l'alimentation risque de soulever de faux biais tout en négligeant les contraintes réelles qui limitent les femmes dans leur accès à d'autres formes de pouvoir d'expression de soi qui dépassent le pouvoir corporel (Thompson 1994, dans Katzman et Lee 1997). 
De façon similaire, une interprétation féministe et transculturelle de la littérature suggère qu'en concevant l'anorexie mentale comme un désordre de l'image corporelle ou un syndrome intrinsèquement lié à la culture occidentale, les modèles qui en dérivent négligent les contextes plus larges et les significations variées du refus de se nourrir (Katzman et Lee 1997). Il importe donc de faire la distinction entre de tels contextes et significations et ceux qui se rattachent aux conditions forcées, comme c'est le cas quand on parle de famine. De même, le recours exagéré à la préoccupation du poids comme variable étiologique de l'anorexie mentale risque d'être indûment ethnocentrique, laissant dans l'ombre d'autres explications plausibles du phénomène (Steiger 1995, dans Katzman et Lee 1997).

Pour sa part, Malson (1997) soulève le manque de théorisation adéquate concernant la relation entre l'individu et la société et plus précisément la manière par laquelle la société est imbriquée dans le problème apparemment individuel de l'anorexie. Ce dernier ne doit pas être conçu comme une pathologie individuelle mais plutôt être compris comme une métaphore et une manifestation de préoccupations et de dilemmes socioculturels particuliers. L'anorexie semble être l'expression de conflits culturels entre la consommation de masse et la minceur normative (Turner 1992, dans Malson 1997), entre les consommateurs indulgents et les travailleurs abstinents et soumis de la société capitaliste (Bordo 1990), et entre les politiques féministes et les idéologies normatives de la féminité (Malson 1997). Bref, Malson (1997) est d'avis que l'anorexie peut être l'expression d'une multitude de conflits et de préoccupations à la fois individuelles et sociétales concernant la féminité, les relations de pouvoir de sexe, la consommation, le contrôle, la rivalité et l'individualisme. Loins d'être le résultat d'un phénomène superficiel de la mode, les désordres alimentaires comme l'anorexie reflètent et demandent donc qu'on se penche sur les maux au coeur de notre culture - à partir de notre héritage historique du dédain pour le corps, en passant par notre peur moderne de perdre le contrôle sur notre avenir, et par la signification alarmante des idéaux contemporains de beauté dans une ère où nous retrouvons pourtant de multiples présences et pouvoirs féminins (Bordo 1988). 
L'image normalisée, la seule «réalité» qui compte, agit dès lors comme un critère contre lequel les autres femmes doivent se mesurer, se juger, se discipliner et se corriger elles-mêmes. Une telle normalisation doit, pour survivre, être constamment mystifiée et effacée dans notre culture par la rhétorique du «choix» et de «'autodétermination» qui jouent un rôle primordial dans les représentations commerciales des régimes amaigrissants, de l'exercice et des autres soins rattachés au corps. La population féminine se voit ainsi placée dans une désolante double contrainte : d'une part, elle est encouragée à se voir elle-même comme «défectueuse» et, d'autre part, elle est châtiée pour ses insécurités (Bordo 1993a).

Bien que les corps soient la propriété des individus, il faut comprendre qu'ils sont définis comme significatifs par la société. Puisque les corps agissent comme agents médiateurs dans la relation entre l'identité personnelle des gens et leur identité sociale, les significations sociales qui sont attachées aux performances et aux formes corporelles particulières ont tendance à être intériorisées et à exercer une forte influence sur le sens que l'individu se donne et les sentiments de valeur intérieure qu'il éprouve (Shilling 1993). La minceur est ainsi valorisée parce qu'elle témoigne du triomphe de l'esprit sur le corps et ses désirs (comme celui de manger). Le corps émacié est produit de manière discursive comme un objet contrôlable, illustrant la perfection et l'intégrité de l'esprit. De manière similaire au corps, le tissu adipeux est vu comme une matière étrangère, une éruption menaçante et excessive. Il s'ensuit, par l'intermédiaire du discours patriarcal et misogyne, la production d'un désir de se détacher complètement de ce corps dénigré, d'où l'émergence des corps anorexiques (Malson 1997).

C'est donc à travers les processus de normalisation et d'intériorisation que nous apprenons à catégoriser les corps et les désirs, de même qu'à les façonner. En attachant une importance esthétique/normative à certains désirs et types de corps, nous favorisons à notre tour le processus de pouvoir. Plus que de simples marionnettes dont on tire les ficelles ou encore de pauvres victimes ignorant les contraintes avec lesquelles elles sont aux prises, les individus sont donc à la fois un effet du pouvoir et l'élément de 
son articulation (Foucault, 1980). En d'autres termes, lorsque le pouvoir s'exerce sur nos connaissances, notre vérité et nos désirs, ce n'est pas simplement quelque chose qui nous est imposé; le pouvoir est également quelque chose que nous intériorisons et dont nous sommes complices dans la production. Nous en venons à désirer les mêmes choses qui limitent nos choix de vie.

Les pratiques disciplinaires auxquelles s'adonne tout particulièrement la population féminine témoignent de la multiplicité des impératifs, des contraintes et des gains potentiels qui agissent sur son désir de se mouler aux idéaux valorisés. Ce regard régulateur et normalisant, qui finit par être intériorisé, peut provenir de différentes sources. Les exemples retenus ont cherché à illustrer comment la santé publique, le système médical et diverses industries exercent ce pouvoir et en tirent profit. Il en résulte une indication claire que l'idéalisation actuelle de la minceur semble être beaucoup plus qu'un reflet de la préoccupation avec la santé ou la mode, ou qu'une simple question d'esthétique.

\section{Conclusion}

Somme toute, le corps véhicule et symbolise toute une série de significations qui viennent à façonner les représentations que l'on se fait de soi-même et des autres. Cette participation à la construction sociale des critères de beauté, qu'elle soit volontaire ou inconsciente, n'est pas sans créer d'importants problèmes. Les pressions grandissantes quant à la poursuite de la minceur et l'adhésion aux images irréalistes diffusées un peu partout risquent d'entraîner des effets dévastateurs, notamment au niveau de l'estime de soi, du développement de l'identité et des perceptions que les individus entretiennent face à leur propre corps. Bien que le développement et la persistance des désordres alimentaires dépendent d'une variété de facteurs, l'emphase de la société sur la minceur, particulièrement chez les femmes, semble être un élément ayant contribué à leur prolifération. Puisque l'insatisfaction de l'image corporelle et l'obsession de la minceur se 
trouvent liées aux désordres alimentaires, une concertation de nos efforts visant à surmonter et à prévenir de telles problématiques est plus que souhaitable. De toute évidence, la société aurait intérêt à élargir ses définitions de la beauté, de manière à ce que chacun et chacune puissent accepter ses différences et les célébrer.

La nature complexe et multifactorielle de l'anorexie suppose ainsi une approche multidisciplinaire. Plutôt que de se heurter à un cadre rigide où symptômalogie et critères diagnostiques priment, il serait peut-être plus bénéfique de veiller à comprendre comment les femmes expriment un conflit qui touche la société à travers leur silhouette et, surtout, d'examiner l'usage que cette société fait du conflit entourant l'image corporelle.

Nul doute, personne ne peut se soustraire aux évaluations d'autrui, la nature humaine étant ce qu'elle est. Toutefois, n'avonsnous pas le droit de vivre sans avoir à nous conformer à des modèles irréalistes, sans avoir à subir des pressions sociales pour y parvenir, sans avoir à croire aux promesses trompeuses qui nous incitent à franchir les canons esthétiques? N'avons-nous pas le droit de nous accepter et d'être acceptées pour qui nous sommes réellement plutôt que pour l'image qui reflète dans la glace, le nombre que renvoie la balance ou le chiffre imprimé sur l'étiquette d'un vêtement? Certes, mais la lutte s'annonce difficile puisque la construction sociale du culte de la minceur, qui s'est érigée par un ensemble de forces, de pratiques et de discours, ne pourra s'écrouler complètement que par un effort collectif. C'est alors, et seulement à ce moment-là, que les actrices sociales pourront être véritablement libérées du poids qui pèse contre elles.

\section{Bibliographie}

AUSTIN, S. Bryn (1999), «Fat, Loathing and Public Health:The Complexity of Science in a Culture of Disordered Eating», Culture, medicine and psychiatry, vol. 2, no 2, 245-268.

BEAULIEU, Ginette (1988), «Le mal-être», dans Musée de la civilisation (sous la dir. du), Souffrir pour être belle, Québec, Éditions Fides, 175-185.

BEAUSOLEIL, Natalie (2000), «Marquage du corps, discipline, résistance et plaisir : les pratiques de maquillage des femmes», dans Sylvie Frigon et Michèle Kérisit (dir. de publ.), Du corps des femmes : contrôles, surveillances et résistances, Ottawa, Les Presses de l'Université d'Ottawa, 231-253. 
BLAIS, Louise (1993), Pauvreté et santé mentale au féminin. L'étrangère à nos portes, Ottawa, Presses de l'Université d'Ottawa.

BORDO, Susan (1988), «Anorexia Nervosa: Psychopathology as the Crystallization of Culture», dans Irene Diamond et Lee Quinby (dir. de publ.), Feminism \& Foucault: Reflections on Resistance, Boston, Northeastern University Press, 87-117.

BORDO, Susan (1989), «The Body and the Reproduction of Feminity: A Feminist Appropriation of Foucault», dans Alison M.Jaggar et Susan R. Bordo (dir. de publ.), Gender/Body/Knowledge: Feminist Reconstructions of Being and Knowing, New Brunswick, NJ, Rutgers University Press, 13-33.

BORDO, Susan (1990), «Reading the Slender Body», dans Mary Jacobus, Evelyn Fox Keller et Sally Shuttleworth (dir. de publ.), Body/Politics:Women and the Discourses of Science, NewYork, Routledge, $83-112$.

BORDO, Susan (1993a), Unbearable Weight: Feminism, Western Culture, and the Body, Berkeley, CA, University of California Press.

BORDO, Susan (1993b), «Feminism, Foucault and the Politics of the Body», dans Caroline Ramazanoglu (dir. de publ.), Up Against Foucault: Explorations of Some Tensions between Foucault and Feminism, Londres, Routledge, 179-202.

BORDO, Susan (1999), Twilight Zones: The Hidden Life of Cultural Images from Plato to O.J., Berkeley, University of California Press.

BOURDIEU, Pierre (1977), Outline of a Theory of Practice, Cambridge, Cambridge University Press.

BOURDIEU, Pierre (1979), La distinction : critique sociale du jugement, Paris, Éditions de Minuit.

DAIGNEAULT-L'AR CHEVÊQUE, Dominique, Lyne DESSUREAULT et Suzanne WALSH (1991), «Un regard féministe sur l'obsession de la minceur», dans Anne Quéniart (dir. de publ.), Femmes et santé: aspects psychosociaux, Boucherville, QC, Gaëtan Morin, 111-131.

DAVIS, Caroline (1997), «Body Image, Exercise, and Eating Behaviors», dans Kenneth R. Fox(dir. de publ.), The Physical Self : From Motivation to Well-Being, Champaign, IL, Human Kinetics, 143-174.

DYRENFORTH, Sue R., Orland W.WOOLEY et Susan C.WOOLEY (1980), «A Woman's Body in a Man's World: A Review of Findings on Body Image and Weight Control», dans Jane Rachel Kaplan (dir. de publ.), AWoman's Conflict:The Special Relationship Between Women and Food, Englewood Cliffs, NJ, Prentice-Hall, 30-57.

FOUCAULT, Michel (1976), Histoire de la sexualité: le souci de soi, 3, Paris, Éditions Gallimard.

FOUCAULT, Michel (1980), Power/Knowledge: Selected Interviews and Other Writings 1972-1977, dans Colin Gordon (dir. de publ.), New York, Pantheon Books.

FRIGON, Sylvie (2000), "Corps, féminité et dangerosité : de la production de "corps dociles" en criminologie», dans Sylvie Frigon et Michèle Kérisit (dir. de publ.), Du corps des femmes : contrôles, surveillances et résistances, Les Presses de l'Université d'Ottawa, Ottawa, 127-164.

GENEST, Françoise (1996), «Barbie, mon miroir et moi», Santé, 115, 60.

GROGAN, Sarah (1999), Body Image : Understanding Body Dissatisfaction in Men, Women and Children, Londres, Routledge.

GROGAN, Sarah et Nicola WAINWRIGHT (1996), «Growing up in the Culture of Slenderness: Girls' Experiences of Body Dissatisfaction", Women's Studies International Forum, vol. 19, no 6, 665-673.

GUÉRICOLAS, Pascale (1998), «Le nouveau culte des apparences: sois belle et battante», La Gazette des femmes, vol. 20, no 1,19-32. 
HESSE-BIBER, Sharlene (1996), Am IThin Enough Yet? The Cult of Thinness and the Commercialization of Identity, New York, NY, Oxford University Press.

ILLICH, Ivan (1992), «Douze mois après la Némisis médicale : Pour une histoire du corps», Dans le miroir du passé : conférences et discours, 1978-1990, Paris, Descartes, 225-263.

JACOBS BRUMBERG, Joan (1997), The Body Project, New York, Random House Inc.

KATZMAN, Melanie A. et Sing LEE (1997), «Beyond Body Image:The Intergration of Feminist and Transcultural Theories in the Understanding of Self Strarvation", International Journal of Eating Disorders, vol. 22, no 4, 385-394.

LAMB, C. Sue, Lee A. JACKSON, Patricia B. CASSIDAY et Doris J. PRIEST (1993), «Body Figure Preferences of Men and Women:A Comparison of Two Generations», Sex Roles, vol. 28, nos 5/6, 345-359.

LE BRETON, David (1997 [1992]), La sociologie du corps, Paris, Presses Universitaires de France.

LESTER, Rebecca J. (1997), «The (Dis)embodied Self in Anorexia Nervosa», Social Science Medicine, vol. 44, no 4, 479-789.

LUPTON, Deborah (1994), Medicine as Culture : Illness, Disease and the Body in Western Societies, Londres, Routledge.

MALSON, Helen (1997), «Anorexic Bodies and the Discursive Production of Feminine Excess», dans Jane M. Ussher (dir. de publ.), Body Talk:The Material and Discursive Regulation of Sexuality, Madness and Reproduction, Londres, Routledge, 223-245.

PALUDI, Michele A. (1998), The Psychology of Women, Upper Saddle River, NJ, Prentice Hall.

POULTON, Terry (1996), No Fat Chicks: How Women Are Brainwashed to Hate Their Bodies and Spend Their Money, Toronto, Key Porter Books.

ROBERTSON, Matra (1992), Starving in the Silences: An Exploration of Anorexia Nervosa, Washington Square, N.Y., New York University Press.

SHILLING, Chris (1993), The Body and Social Theory, Londres, Sage Publications.

SPARKES, Andrew (1997), «Reflections on the Socially Constructed Physical Self», dans Kenneth R. Fox (dir. de publ.), The Physical Self: From Motivation to Well-Being, Human Kinetics, Champaign, IL, 83-110.

SPITZACK, Carole (1990), Confessing Excess:Women and the Politics of Body Reduction, Albany, NY, State University of New York Press.

TRAVAILLOT,Yves (1998), Sociologie des pratiques d'entretien du corps : l'évolution de l'attention portée au corps depuis 1960, Paris, Presses Universitaires de France.

WEISS, Gail (1999), Body Images: Embodiment as Intercorporeality, New York, Routledge.

WOLF, Naomi (1990), The Beauty Myth, Toronto, Random House.

ZOLA, Irving (1992), "Culte de la santé et méfaits de la médicalisation», dans Luciano Bozzini, Marc Renaud, Dominique Gaucher et Jaime Llambias-Wolff (dir. de publ.), Médecine et société : les années 80, Montréal, Éditions Coopératives Albert Saint-Martin, 31-51.

\section{Note}

1. L'article s'appuie sur le mémoire Disciplinarisation des corps féminins et poursuite de l'idéal de la minceur : des pratiques lourdes de sens (2000) rédigé par l'auteure, sous la direction de Louise Blais de l'Université d'Ottawa. 